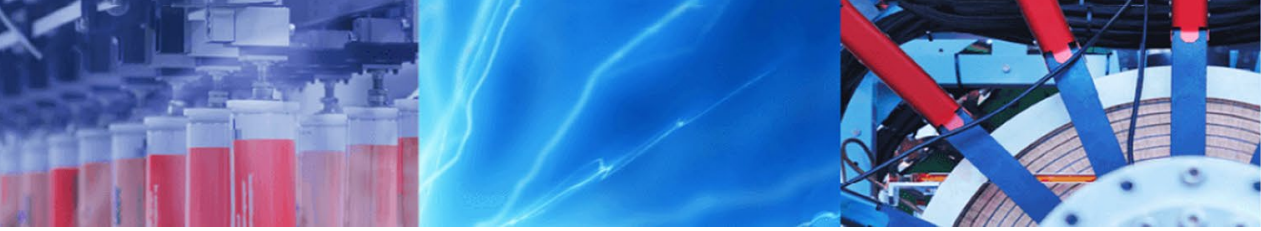

Research Article

\title{
Experimental investigation of porous medium structural effects on a coupled porous media-free zone laminar flow
}

\author{
J. K. Arthur ${ }^{1}$ \\ (c) Springer Nature Switzerland AG 2019
}

\begin{abstract}
An experimental research program was conducted to investigate the effects of solid volume fraction, arrangement of rods, and rod shape on slow flow through and over two-dimensional model porous media. The porous media were modeled using in-line and staggered arrays of rods of circular and square cross-sections to cover a range of solid volume fraction of 0.06 to 0.49 . Each model was installed into a test section so as to fill three-quarters of the total depth of the flow section; and the flow was driven through the test section by pressure. Using a refractive-index matched viscous fluid, the bulk Reynolds numbers for the respective test conditions were kept considerably lower than unity. Particle image velocimetry measurements were made across the streamwise-transverse plane of the test section. Results indicate that the flow through the free zone increases from $75 \%$ to nearly $100 \%$ as the solid volume fraction increases from 0.06 to 0.49. While the bulk distribution is independent of the shape of the rods, or the mode of arrangement, at the interface, penetration of the free flow is dependent on the solid volume fraction and arrangement of rods. The interfacial flow is predicted by a new formulation from which a boundary condition is derived which unlike others in the literature, is devoid of jumps at the interface. This work provides detailed experimental data and important predictive tools for validations of theoretical and numerical work.
\end{abstract}

Keywords Particle image velocimetry · Porous medium · Interfacial flow · Boundary condition

\section{Introduction}

The study of steady, single-phase and incompressible laminar flow through and over porous media has an essential bearing on engineering applications such as river bed flows, permeable reactive barrier technology, packed bed heat exchanger insulations, and chemical drying devices. This has been the subject of extensive research, as reviewed in previous publications [1, 2]. Figure 1 illustrates such a coupled flow problem.

To analyse flow through and over porous medium, two distinct levels of descriptions are considered, namely the microscopic and averaged descriptions. For the microscopic description, microscopic momentum equations such as the Navier-Stokes or Stokes equation are used to describe the entire flow section. Although this description generally leads to a detailed study of the physics of the flow, due to prohibitive computational costs of simulating the porous medium flow, it is not applicable for most practical cases [3].

In the averaged description, the flow in the porous medium is always described by an averaged equation such as the Darcy law while the free zone is also described by a microscopic or averaged form of the Navier-Stokes or Stokes equation. Although the averaged description somewhat simplifies the flow description within the porous medium, the solution of the problem is still a challenge. This is because it involves the matching of governing equations at the nominal interface between the porous medium and the non-porous medium (i.e. the free zone). As the equations of the flow domains are of different orders, the use of a boundary equation to match

J. K. Arthur, james.arthur@bucknell.edu | 'Department of Mechanical Engineering, Bucknell University, Lewisburg, PA, USA.

SN Applied Sciences (2019) 1:1062 | https://doi.org/10.1007/s42452-019-1070-7 
Fig. 1 Flow through and over a porous medium. A typical streamwise velocity profile for a given streamwise location in a pressure-driven flow

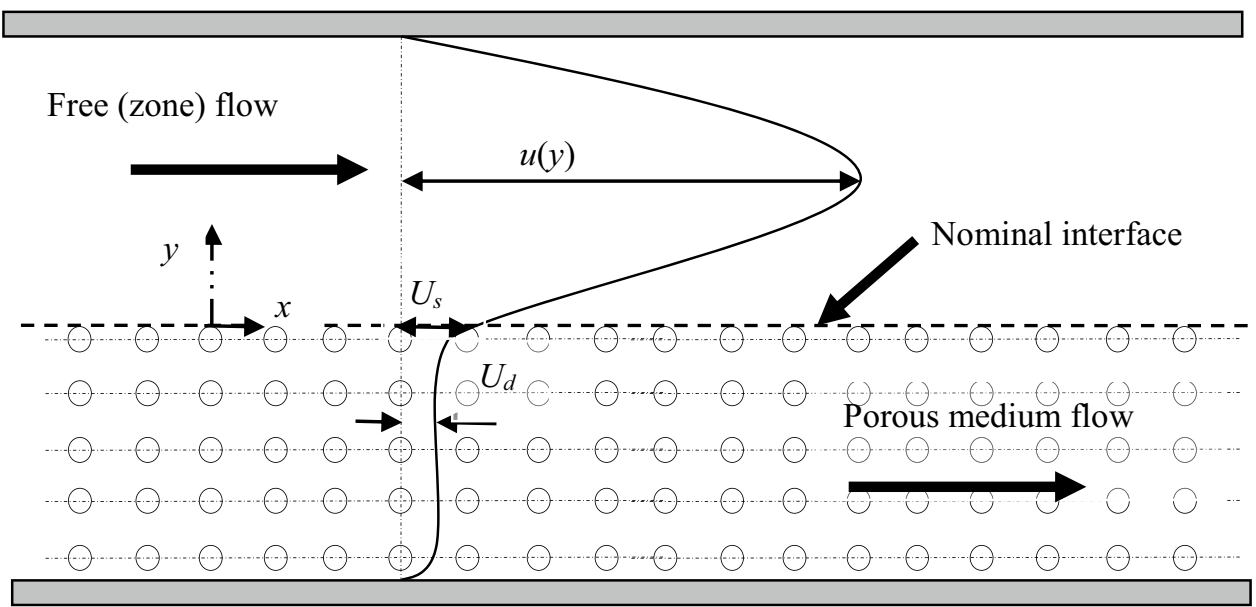

them becomes necessary. However, because the interfacial momentum transfer at the interface is complicated in nature, this boundary condition is difficult to define.

To address these issues, the following approaches are commonly adopted. For the specific case of coupled flow domains governed by the Darcy law and Stokes equation for example, one approach is to use a macroscopic Brinkman equation [4] for the averaged flow in lieu of the Darcy law and Stokes equation. For a flow of dynamic viscosity $\mu$, averaged streamwise velocity $U$, streamwise pressure gradient $d P / d x$ across an isotropic porous media sample of specific permeability $k$, the Brinkman equation may be expressed as

$-\frac{d P}{d x}+\mu^{\prime} \nabla^{2} U=\frac{\mu U}{k}$

While the Brinkman equation is widely used in the literature, there seems to be no consensus as to how the so-called apparent viscosity $\mu^{\prime}$ is to be modelled [5-7]. This makes this method of solution an approximation at best, needing further experimental verification. A second approach to solving the coupled Stokes and Darcy coupled flow problem is to use an interfacial boundary condition such as the one proposed by Beavers and Joseph [8] (hereafter called the BJC for convenience). This condition, in terms of Fig. 1 may be expressed as

$\left.\frac{d u}{d y}\right|_{y=0-}=\frac{\alpha}{k^{0.5}}\left(U_{s}-U_{d}\right)$

where $d u /\left.d y\right|_{y=0 \text { - }}$ is the interfacial microscopic shear rate in the free zone, $U_{s}$ is the average streamwise velocity at the interface (i.e. the slip velocity), $U_{d}$ is the uniform average streamwise velocity within the porous medium and $a$ is an empirical slip coefficient. The limitation of the BJC however, is that $a$ is scarcely provided in the literature. Additionally, the use of the BJC leads to a jump in both velocity and stress at the interface, so that the portion of the velocity profile from $U_{s}$ to $U_{d}$ (i.e., the transition layer within the porous medium) is not well accounted for. Other interfacial boundary conditions have also been proposed and explored. One of such is the boundary condition by Ochoa-Tapia and Whitaker [9] (hereafter called OWC for convenience). For a porous medium of porosity $\varepsilon$, this condition can be expressed as:

$\left.\frac{1}{\varepsilon} \frac{d U}{d y}\right|_{y=0-}-\left.\frac{d U}{d y}\right|_{y=0+}=\frac{\beta}{k^{0.5}} U_{s}$

In Eq. (3), $d U /\left.d y\right|_{y=0+}$ and $d U /\left.d y\right|_{y=0 \text { - }}$ are respectively average interfacial shear rates in the porous medium and in the free zone; and $\beta$ is a coefficient that needs experimental determination. It should be noted that while the OWC has been found to be of great help as an analytical tool, its utility is limited because $\beta$ is also not adequately given in the literature. Furthermore, the use of the OWC necessitates a velocity gradient discontinuity at the interface, which does not apply to all cases of the problem described in Fig. 1. These problems are similar to that of other conditions (e.g. Chandesris and Jamet [3]) proposed employing similar methods as used in obtaining the OWC. This leaves the problem of laminar flow over porous media an open one that needs to be addressed experimentally.

Previous studies however indicate that the interfacial flow is rather complicated and dependent on a number of "structural" factors such as the solid volume fraction, type of the porous medium, and filling fraction of the porous medium flow [10]. These factors ought to be characterized if the interfacial and transition layer flow is to be properly understood and predicted. Conversely, experimental data of such kind is rather limited in the literature. It is based on this background that this research program has been designed to investigate pressure-driven laminar flows through and over arrays of model porous media. 
In this work, attention is focused on velocity measurements of slow flow through and over porous media. Twodimensional regular arrays of rods are used to model real porous media. The literature contains studies of velocity measurements of random porous media [11]. However, the two-dimensional model as explored in this study, is still appropriate [12] and relevant for this study because of its practical utility to provide valuable insight into flow phenomena that may be applied in real cases such as banks of heat exchanger tubes and fibrous porous media. It should be noted that this model has been used in previous numerical $[5,6,10]$ analytical [13], and experimental studies [14-16]. However, this work presents a rarely published experimentally tested case of pressure-driven flow through such a model medium [16].

The objectives of the present paper then are mainly twofold. The first objective is to provide velocity measurements of slow flow through and over model porous media, and to use the averaged measurements to characterize the effects of solid volume fraction, arrangement of rods, and rod shape on the flow. The second objective is to use the averaged measurements to verify formulations that have been proposed in the literature with the view to proposing formulations that will serve as better predictive tools in describing the interfacial flow. These goals are achieved using twodimensional porous media of solid volume fraction ranging from 0.06 to 0.49 , so that a wide range of loosely packed and compact porous media is covered. The mode of arrangement of porous media rods is also tested using in-line and staggered arrays; and the shape of the cross-sectional area of the rods also tested using rods of porous media with circular and square cross-sectional shapes. The filling fraction of the models, and the bulk Reynolds numbers for the respective test conditions are kept constant so that filling fraction and inertial effects are not relevant. Detail velocity measurements are obtained using a high-resolution particle image velocimetry (PIV) technique in a refractive-index matched viscous fluid-flow section. A major contribution of this work is that the averaged measurements are used to provide a new formulation to predict the interfacial flow at a reasonable level of accuracy. As this work is based on data obtained in a larger experimental doctoral research program reported as Arthur [17], a much more comprehensive information on the data may be referenced from that doctoral dissertation.

\section{Experimental set up and measurement procedure}

The experiments were conducted in a transparent acrylic channel of length $500 \mathrm{~mm}$, span $115.5 \mathrm{~mm}$ and depth 115.5, as shown in Fig. 2. The channel was constructed from a transparent acrylic sheet of refractive index (RI)
1.47 , so as to allow optical access. It was designed so as to let flow into it at the upstream end, then through a $200 \mathrm{~mm}$ section reserved for flow conditioning, and finally through a $300 \mathrm{~mm}$ test section in a reasonably uniform flow, before exiting in another central exit hole at the downstream end.

The porous media models are also schematically shown in Fig. 2b, c. Each model was constructed from two side plates, one lower plate and rods. The plates and rods were all made from optically clear acrylic material of $\mathrm{RI}=1.47$. In the construction, holes were drilled through the side plates so that circular rods of diameter $d$ or square rods of side $s$ could be inserted into them with the axes of the rods aligned in the spanwise direction. The rods were spaced at a distance / determined from the relations:

$I=d / 2(\pi / \phi)^{0.5}$

$I=s / \phi^{0.5}$

Equation (4) was used for porous media array of circular rods, whilst Eq. (5) was used for that of square rods. Circular rods of diameters $d=3.18 \mathrm{~mm}$ and $4.76 \mathrm{~mm}$, and square rods of side $s=3.18 \mathrm{~mm}$ were used. The rods of the models were mounted in square arrays, yielding solid volume fractions within the range $0.06 \leq \phi \leq 0.49$

Five models were of in-line arrangements of rods (as shown in Fig. 2b). One of the models was of staggered arrangement. The latter model is hereafter referred to the "staggered model" to distinguish it from models of in-line array of rods which are also henceforth called non-staggered models. In the staggered model, the holes were arrayed as shown in Fig. 2c, so that alternate columns of the porous media models were staggered by a distance of $I / 2$ along the stream. Each of the porous media model types (whether staggered or non-staggered) was designed to cover a minimum of ten rows of rods. When fully assembled, each porous medium model consisted of three plates screwed together to form a box of length $300 \mathrm{~mm}$, span $109.5 \mathrm{~mm}$, and a total depth $H=109.5 \mathrm{~mm}$. With a porous medium depth $h=82.1 \mathrm{~mm}$, models of filling fraction $h / H=0.75$ was achieved. The porous media models were tested by placing them into the measurement section of the test channel as shown in Fig. $2 \mathrm{~d}$.

The coordinate system used in this work is also indicated in Fig. 2 for an installed model in the test channel. The origin of the streamwise direction $x=0$ was coincident with the center of the most upstream column of rods, which is also $230 \mathrm{~mm}$ downstream from the inlet of the test channel. For the transverse direction, its origin $Y=0$ was located at the bottom wall, and $y=0$ was located at the edge of the porous media in closest proximity with the free zone. The origin of the spanwise direction $z=0$ was fixed at the mid-span of the channel. 
Fig. 2 a A schematic of the test channel. The shaded section is to distinguish the flow conditioning section from the measurement section. Porous media models in: $\mathbf{b}$ front section showing nonstaggered array $\mathbf{c}$ front section showing staggered array d three-dimensional view, as installed in the test channel. The coordinate directions used in the experiments are shown. All numeric dimensions are in millimetres

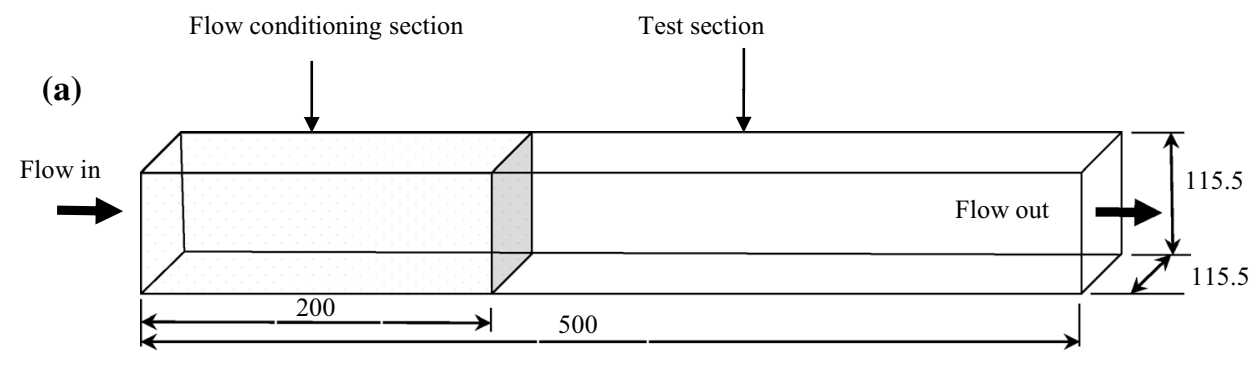

(b)

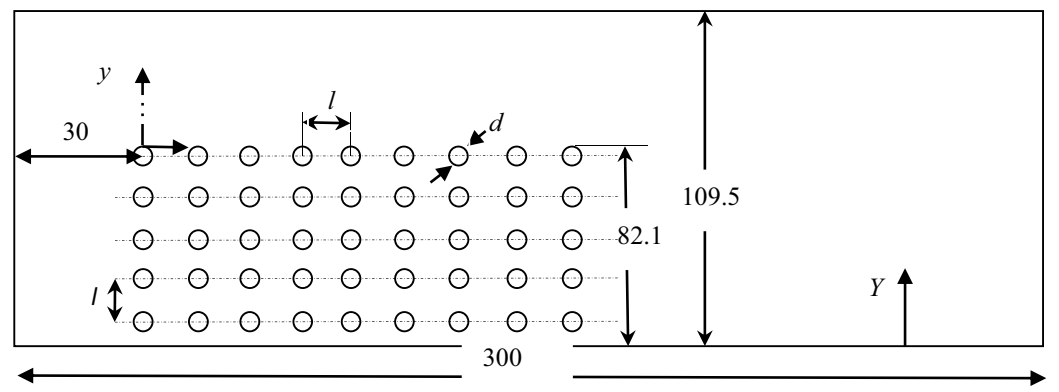

(c)

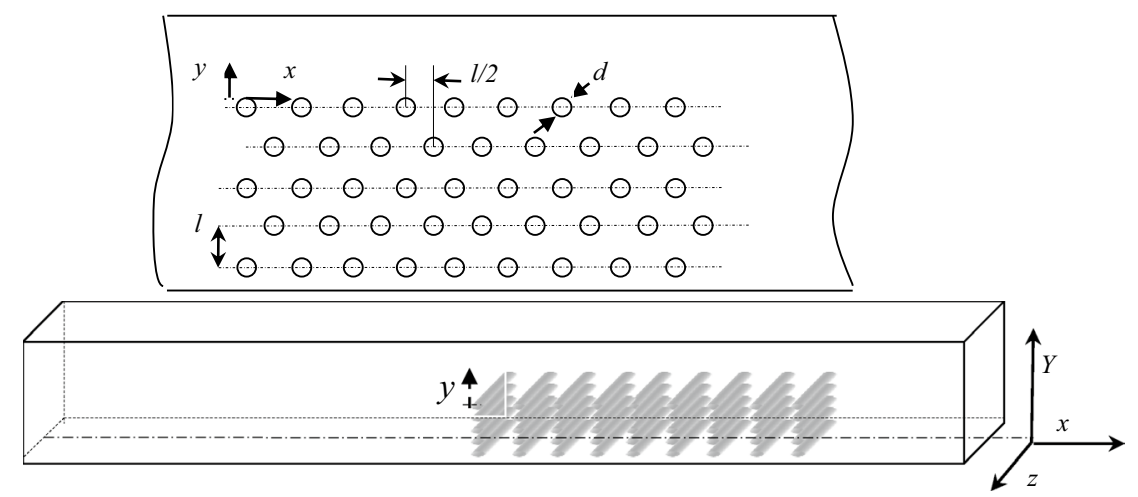

A planar PIV system was used for the velocity data acquisition. The hardware for the PIV system is detailed in an earlier work [17]. A Dantec Dynamic DynamicStudio commercial software installed on an Intel(R) Core computer was used to operate the PIV system, and to process data that was acquired. The working fluid was a Cargille Immersion liquid (Code 5040) of kinematic viscosity $v=20 \times 10^{-6} \mathrm{~m}^{2} / \mathrm{s}$ (at $25^{\circ} \mathrm{C}$ ), density $\rho=848 \mathrm{~kg} / \mathrm{m}^{3}$ and $\mathrm{RI}=1.47$. This fluid was seeded with silver-coated hollow glass spheres of mean diameter $10 \mu \mathrm{m}$ and specific gravity 1.4. Based on the working fluid and seeding particles, the particle settling velocity and response time were estimated to be $1.77 \mu \mathrm{m} / \mathrm{s}$ and $7.98 \mathrm{ps}$, respectively. As these values are very small compared with the typical velocity and time scales used in the experiment, the particles were considered to follow the fluid faithfully.

As schematized in Fig. 3, the PIV measurements were performed in the streamwise-transverse plane of the test channel. Optimum background contrast and resolution were kept by using requisite camera focal lengths. By ensuring that particle image diameters were of 2.3 pixels (which is close to 2 pixels as recommended by Raffel [18]), errors due to peak-locking were kept minimal. In order to ensure a good signal to noise ratio, images were acquired using laser pulses, timed in such a way that the particle displacement in an interrogation area (IA) was less than a quarter of the side of the IA [19]. In the present experiments, using an interrogation window of size recording of 32 pixels by 32 pixels, and a sub-pixel accuracy of 0.1 the dynamic range is estimated to be 80 [20]. The field of view was $27 \mathrm{~mm}$ per side, and the scale factor was $\sim 1.8$. The resultant spatial resolution was about $0.42 \mathrm{~mm}$. By maintaining an overlap of $50 \%$ between neighbouring interrogation areas during the processing of data, additional vectors were provided so that the distance between neighbouring vectors were $\sim 0.21 \mathrm{~mm}$. Optimal vector correlations were obtained by post-processing particle images using the adaptive-correlation option of the DynamicStudio v.2.30 software. Extensive velocity measurements were conducted with the planar PIV per various test conditions along various $z$ planes.

In this work, all microscopic quantities are denominated by italicized lower case letters, and their corresponding averaged quantities by italicized upper case letters. For 
Fig. 3 A schematic of PIV arrangement with test channel and test models

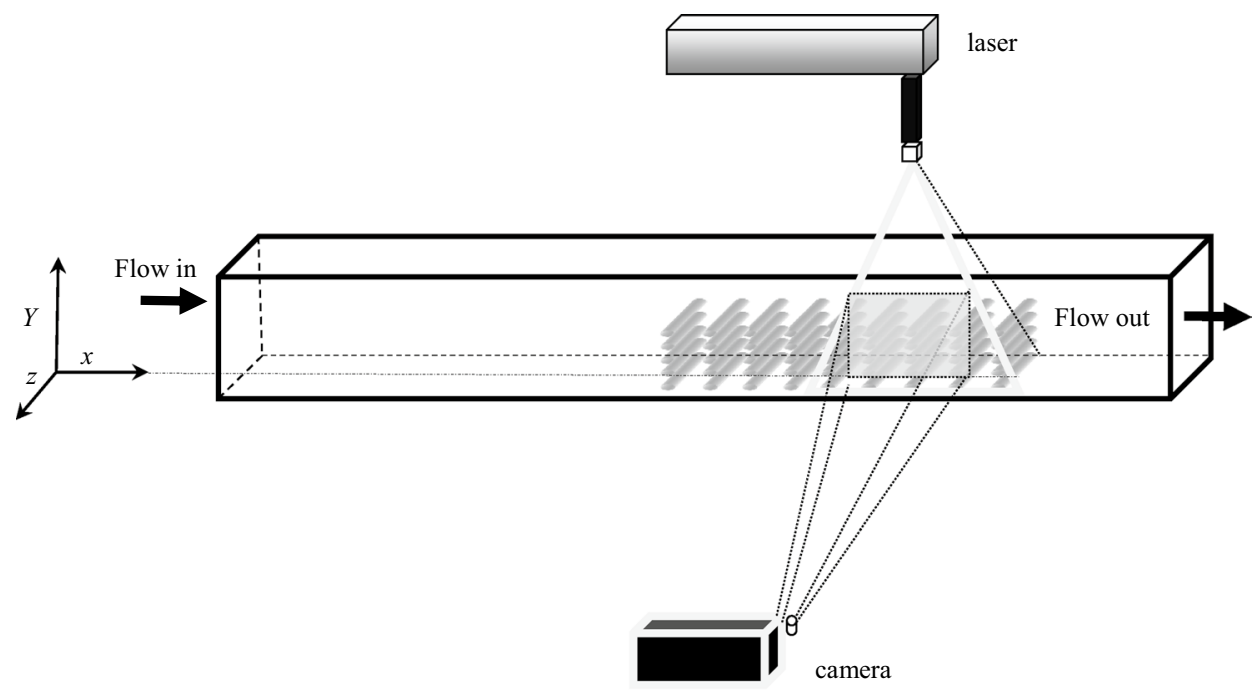

the Cartesian frame of reference, the components of the microscopic velocity in the streamwise $(x)$ and transverse (y) directions are designated respectively by $u, v$. Averaged velocities in the $x, y$ directions are similarly denoted respectively by $U, V$. To study the variation of the averaged streamwise velocities between rod centers, volume averaged streamwise and transverse velocities were computed respectively as follows

$U=U_{v}=\frac{1}{\beta^{3}} \int_{0}^{1} \int_{0}^{l} \int_{0}^{1} u(x, y, z) d x d y d z$

$V=V_{v}=\frac{1}{\beta^{3}} \int_{0}^{l} \int_{0}^{l} \int_{0}^{l} v(x, y, z) d x d y d z$

A number of preliminary experiments were carried out. The first of those experiments were done to assess the sample size $N$ necessary to achieve statistical convergence. As shown in Fig. 4a, velocity measurements were found to be independent of the sample size for $N \geq 10$. Hence, a minimum sample size of $\mathrm{N}=30$ images was used in subsequent measurements.

The accuracy of the PIV system was also verified (Fig. 4b), using fully developed velocity profiles obtained for a plane laminar channel flow. An assessment of the velocity measurement uncertainties was also undertaken. This was based on standard methodology [21,22], and for fifteen rounds of experiments. The uncertainty in streamwise velocity $u$ in the free zone is approximately $1 \%$ of the local maximum velocity, $u_{\max }$. For model porous media of $\phi=0.06,0.12,0.22$ and 0.49 , the uncertainties of u measured for flows through them are approximately $1.5 \%, 2.5 \%, 4 \%$ and $5 \%$ respectively of $\mathrm{u}_{\max }$ due to relatively low optical assess and lower velocities measured through these models. For the transverse velocities $v$, total uncertainties are also estimated to be $0.3 \%$ of $\mathrm{u}_{\max }$ in the free zone; and $0.5 \%, 2 \%, 3 \%$ and $3.5 \%$ of $\mathrm{u}_{\max }$ in models $\phi=0.06,0.12,0.22$ and 0.49 respectively. All error estimates are at $95 \%$ confidence level, and are signified by error bars in pertinent plots in this work.

Previous works have established that after a number of rows of rods, the flow through the porous medium becomes periodic [23]. To determine this region of periodicity, measurements were made in the $x-y$ plane at $z=0$, and at various flow rates and with various models. Results were extracted at various locations along the transverse direction, and compared, and it was found that the flow generally became periodic at $\mathrm{x} / \mathrm{l}>3$, as shown in Fig. 4c. Thus, all subsequent analyses were done using measurements at $x / l \geq 4$.

Through testing, it was demonstrated that the spanwise variations of porous medium flow were negligible at least within $-15 \mathrm{~mm}<\mathrm{z}<15 \mathrm{~mm}$ (which is greater than the size of a unit cell for the smallest solid volume fraction, $\phi$ ). This is shown in Fig. 4d. Thus, it was not necessary to take velocity measurements along multiple sections in the spanwise direction. Succeeding measurements were therefore conducted only in the $z=0$ plane.

Further tests were performed, as summarized in Table 1. In the table, the shape of the rod cross-sectional area, rod arrangement, $d, s, l$, and $\phi$ of the test models per condition are shown. The streamwise bulk velocity $\mathrm{U}_{\text {bulk }}$ was calculated from

$U_{\text {bulk }}=\frac{1}{H} \int_{0}^{H} U d Y$

The characteristic bulk Reynolds number was computed from 
Fig. 4 a Sample of convergence test using ensembleaveraged streamwise $(\mathrm{u})$ measurements of flow through non-staggered model of $\phi=0.22$, and taken at $z=0$ and extracted at $x / I=6.5$. $\mathbf{b}$ Verifying velocity measurements using fully developed channel flow $u$ profiles taken at $z=0$. cSample results of data measurements of tests with non-staggered test model of $\phi=0.06$ taken at $z=0$ and extracted at $y / I=-1.6$ to show flow periodicity at $x / I>3$. d Measurements in non-staggered test model of $\phi=0.06$ at $\mathrm{x} / \mathrm{l}=4.5$ showing that at least within $-15 \mathrm{~mm}<\mathrm{z}<15 \mathrm{~mm}$, the flow variation was negligibly small for flow within the porous medium, and near the interface
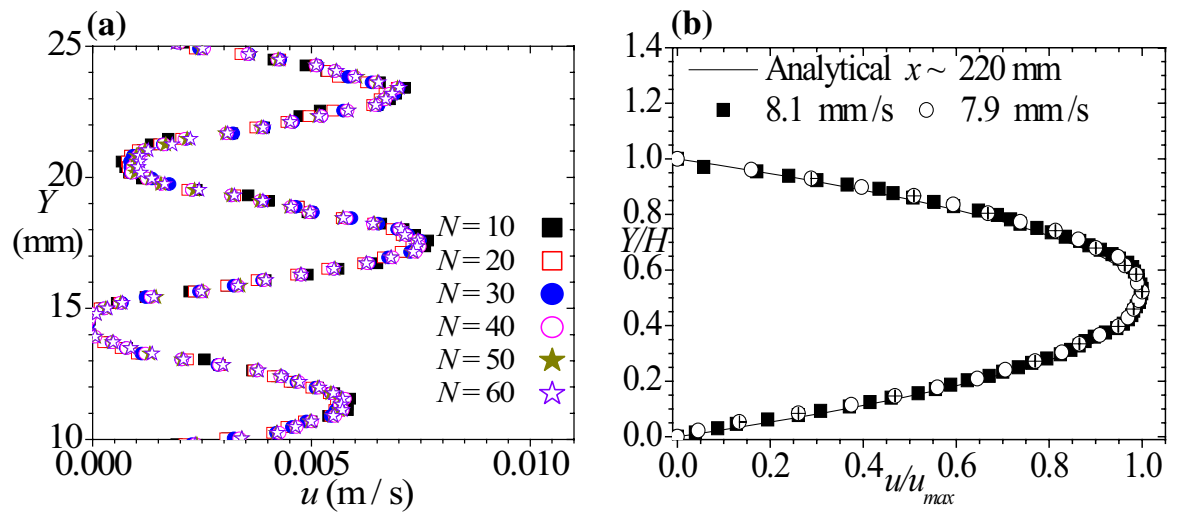

(c)

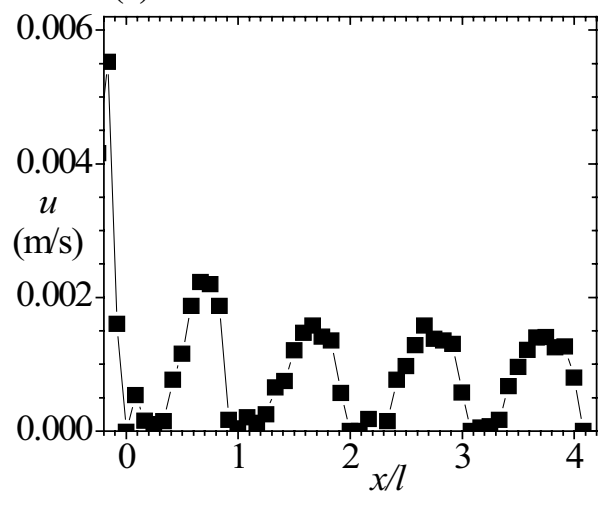

(d)

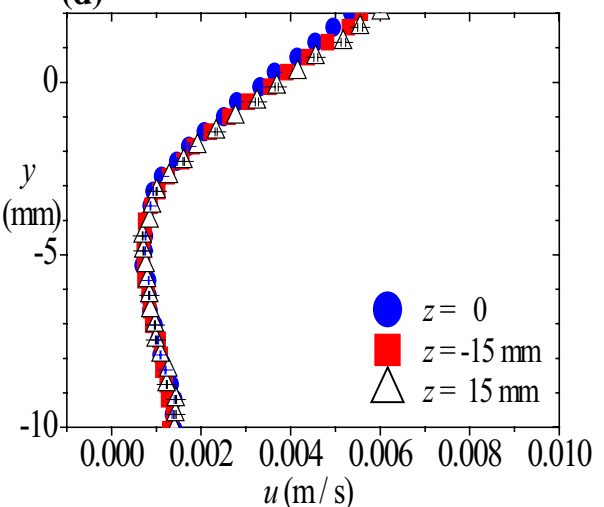

Table 1 Summary of Test Conditions

\begin{tabular}{|c|c|c|c|c|c|c|c|}
\hline $\begin{array}{l}\text { Shape of rod } \\
\text { cross section }\end{array}$ & Arrangement & $\mathrm{d}$ or $\mathrm{s}(\mathrm{mm})$ & $\mathrm{I}(\mathrm{mm})$ & $\phi$ & $\mathrm{U}_{\text {bulk }}(\mathrm{mm} / \mathrm{s})$ & $\mathrm{Re}_{\text {bulk }}$ & $\begin{array}{l}\text { Name of } \\
\text { test condi- } \\
\text { tion }\end{array}$ \\
\hline Round & Non-staggered & 3.18 & 12 & 0.06 & 1.6 & 0.3 & $\mathrm{RdN} \phi_{6}$ \\
\hline Round & Non-staggered & 3.18 & 8 & 0.12 & 1.9 & 0.3 & $\operatorname{RdN} \phi_{12}$ \\
\hline Round & Non-staggered & 3.18 & 6 & 0.22 & 1.7 & 0.3 & $\operatorname{RdN} \phi_{22}$ \\
\hline Round & Non-staggered & 4.76 & 6 & 0.49 & 1.1 & 0.3 & $\operatorname{RdN} \phi_{49}$ \\
\hline Square & Non-staggered & 3.18 & 9 & 0.12 & 1.6 & 0.3 & $\mathrm{SqN} \phi_{12}$ \\
\hline Round & Staggered & 3.18 & 8 & 0.12 & 1.9 & 0.3 & $\mathrm{RdSt} \phi_{12}$ \\
\hline
\end{tabular}

$R e_{\text {bulk }}=U_{\text {bulk }} d / v=0.3$

$R e_{\text {bulk }}=U_{\text {bulk }} s / v=0.3$

With the $\mathrm{Re}_{\text {bulk }}$ kept constant, inertia was considered not to be a factor. Each of the test conditions are denominated as shown in the table for convenience.

\section{Results and discussion}

In this section, the results of experiments are presented and discussed by first examining the bulk flow, and then focusing on the flow at the interface of the porous medium, and finally on the prediction of interfacial flow. This discussion is largely limited to volume averaged results as such results provide a sufficiently representative view of the averaged flow.

\subsection{Bulk flow characterization}

In Fig. 5, results are presented to show the relative magnitudes of volume averaged streamwise $\left(U_{v}\right)$ and transverse $\left(V_{v}\right)$ velocity data, non-dimensionalized by the maximum streamwise velocity in the free zone $U_{v \text {, max }}$ in the same figure. In all cases, the $V_{v}$ is no more than $3.5 \%$ of $U_{v, m a x}$. Indeed, the relative proportion of $V_{v}$ with respect to $U_{v \text {, } \max }$ was largely within error bounds, and could therefore 
be neglected. Thus, $U_{v}$ should be the subject of greater attention.

Solid volume fraction effects are shown in Fig. 6 by the plots of the percentage of the total mass flow through the test section that is channelled through the free zone (Fig. 6a), and the ratio of the maximum velocity to bulk velocity, $U_{v, \text { max }} / U_{\text {bulk }}$ (Fig. $6 \mathrm{~b}$ ). The results are further summarized in Table 2. Present results show that the proportion of flow channelled into the free zone increases from 76 to $98 \%$ as $\phi$ increases from 0.06 to 0.49 . This is because the proportion of flow conducted through the porous medium reduces with increase in $\phi$ due to an increased resistance. This trend also follows that observed in an earlier work [24] using three-dimensional porous media configurations in which rod axes were arrayed in the transverse direction. It follows then that the increase in percentage flow through the free zone when $\phi$ increases occurs independent of the type of porous medium. Figure 6 and Table 2 also show that as $\phi$ increases, $U_{v, \max } / U_{\text {bulk }}$ increases, tending towards the limiting value of 6 expected for the case where the porous media is replaced by a solid block. The $U_{v, \max } /$ $\mathrm{U}_{\text {bulk }}$ trends of variation in $\phi$ are similar to measurements done for other models in [24].
Fig. 5 The relative magnitudes of volume averaged streamwise $\left(\mathrm{U}_{\mathrm{v}}\right)$ and transverse $\left(\mathrm{V}_{\mathrm{v}}\right)$ average velocities indicated in volume averaged plots for the flow through and over the porous media

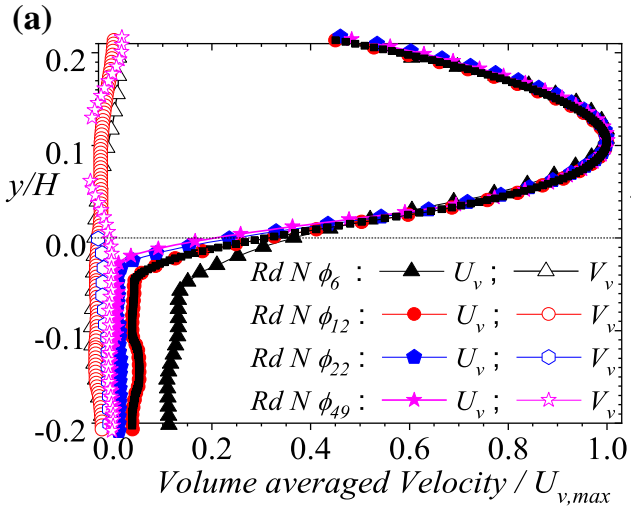

(b)

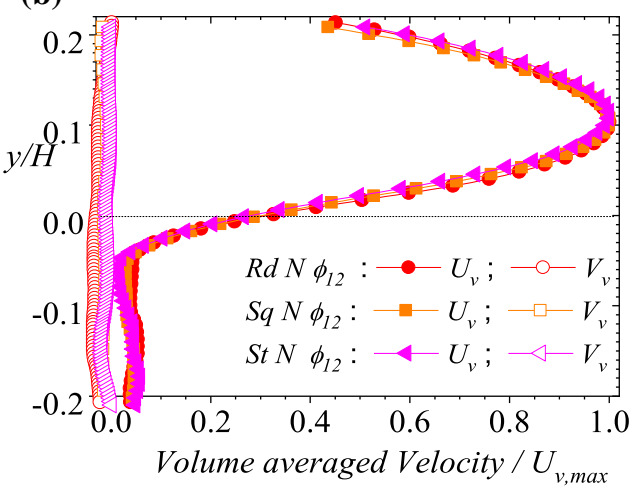

Fig. 6 a Percentage flow channelled in the free zone; and $\mathbf{b}$ $\mathrm{U}_{\mathrm{v}, \max } / \mathrm{U}_{\text {bulk }}$ ratios for the test conditions (a)

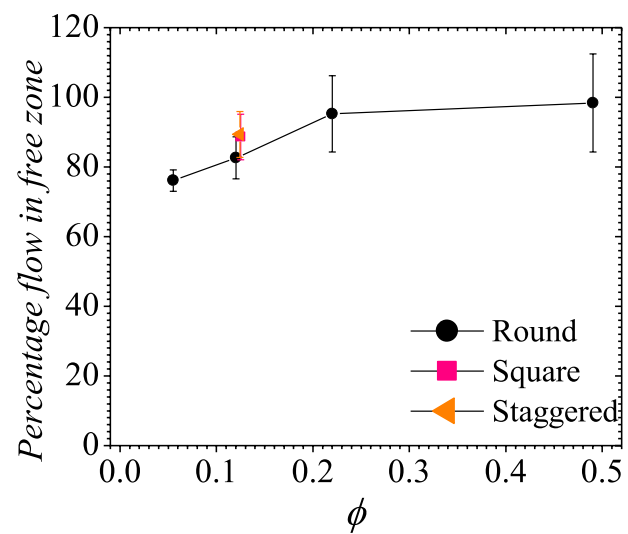

(b)

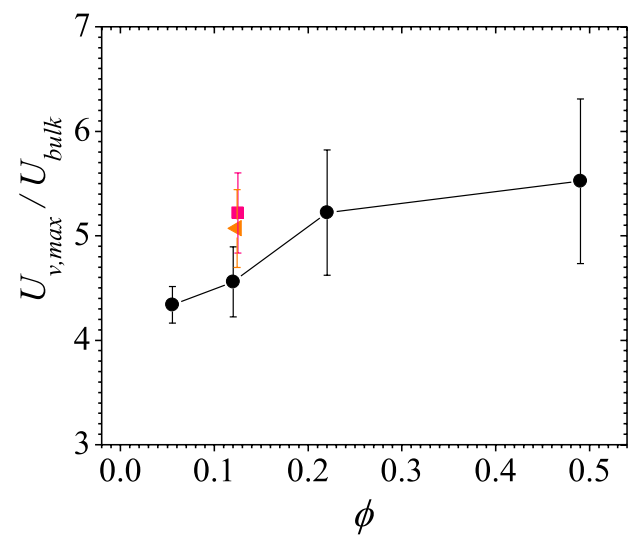

Table 2 Summary of results of volume averages

\begin{tabular}{|c|c|c|c|c|c|c|c|c|c|}
\hline Name of test & $\begin{array}{l}\text { Flow in free } \\
\text { zone (\%) }\end{array}$ & $\mathrm{k}^{0.5}(\mathrm{~mm})$ & $\mathrm{U}_{\mathrm{v}, \mathrm{s}}(\mathrm{mm} / \mathrm{s})$ & $\dot{\gamma}_{v}(/ \mathrm{s})$ & $\mathrm{U}_{\mathrm{v}^{\prime} \text { max }} / \mathrm{U}_{\text {bulk }}$ & $\mathrm{U}_{\mathrm{ars}} / \mathrm{U}_{\mathrm{v}, \max }$ & $\mathrm{U}_{\mathrm{v}, \mathrm{S}} \mathrm{S}\left(\dot{\gamma}_{\mathrm{v}}\right)$ & $\mathrm{U}_{\mathrm{v}, \mathrm{s}} /\left(\dot{\gamma}_{v} k^{0.5}\right)$ & $\mathrm{U}_{\mathrm{v}, \mathrm{s}} /\left(\dot{\gamma}_{v} I_{s}\right)$ \\
\hline $\operatorname{RdN} \phi_{6}$ & 76 & 3.0 & 2.4 & 0.40 & 4.07 & 0.36 & 5.87 & 1.98 & 2.94 \\
\hline $\operatorname{RdN} \phi_{12}$ & 83 & 1.4 & 2.6 & 0.79 & 4.47 & 0.31 & 3.33 & 2.38 & 2.50 \\
\hline $\operatorname{RdN} \phi_{22}$ & 95 & 0.8 & 2.2 & 0.92 & 5.18 & 0.24 & 2.34 & 3.00 & 2.37 \\
\hline $\operatorname{RdN} \phi_{49}$ & 98 & 0.3 & 1.2 & 0.60 & 5.43 & 0.21 & 2.00 & 7.74 & 2.36 \\
\hline $\mathrm{SqN} \phi_{12}$ & 89 & 1.5 & 2.4 & 0.68 & 4.96 & 0.30 & 3.56 & 2.45 & 2.34 \\
\hline RdSt $\phi_{12}$ & 89 & 0.9 & 2.5 & 0.80 & 4.92 & 0.27 & 2.81 & 3.40 & 2.00 \\
\hline
\end{tabular}


The effects of the rod shape (i.e., circular and square) and arrangement (i.e., non-staggered and staggered) on the bulk flow may also be observed from Fig. 6. It may be noted that the bulk flow appears to be independent of these factors. The percentage flow channelled into the free zone remains at $\sim 87 \%$ for non-staggered, staggered models of $\phi=0.12$. The ratios $\mathrm{U}_{\mathrm{v}, \max } / \mathrm{U}_{\text {bulk }}$ are also nearly unaffected by the rod shape or arrangement.

\subsection{Interfacial flow characterization}

To characterize the interfacial flow, the dimensionless realizations of the specific permeability $k$, the channel's maximum velocity $U_{v \text {, max }}$ the seepage velocity $U_{d}$, the slip velocity $\mathrm{U}_{\mathrm{v}, s^{\prime}}$ and shear rate at the interface $\dot{\gamma}_{\mathrm{v}}\left(=\mathrm{dU}_{\mathrm{v}} /\right.$ $\left.d y\right|_{y=0}$ ) were studied. The specific permeabilities of the test models were quantified using correlations provided in the literature. For models of rods of radius $r$, distance between rod centers $I$, the following relations $[10,25,26]$ were used for solid volume fractions $\phi$

$$
\begin{aligned}
k= & \frac{r^{2}}{8 \phi}\left(-\ln \phi-1.476+2 \phi-1.774 \phi^{2}+4.076 \phi^{2}\right) \\
k= & 0.0602(1-\phi)^{5.1} l^{2} \\
k= & \frac{\pi r^{2}}{\phi}\left(0.0406(1-\phi)^{2.5}-0.2356(1-\phi)^{3.5}+0.3422(1-\phi)^{4.5}\right. \\
& \left.+0.5960(1-\phi)^{5.5}-1.5601(1-\phi)^{6.5}+0.9092(1-\phi)^{2.5}\right)
\end{aligned}
$$

Equation (11) was used for non-staggered models of $\phi=0.06$, and Eq. (12) was used for non-staggered models of $\phi \geq 0.12$. Equation (13) was also used for staggered model. Values of $\mathrm{k}^{0.5}$ are provided in Table 2 . The velocity $U_{d}$ for each test condition was also obtained by averaging the streamwise velocities only over a distance of about $-0.1 \lesssim y / H \lesssim-0.3$. The particular range for each test condition covered was modified depending on $\phi$ so as to shield the $U_{d}$ from any errors emanating velocities close to the interface and the lower wall boundary layer. Following previous related works $[10,27]$, the slip velocity $\mathrm{U}_{\mathrm{s}}$ is defined as the average streamwise velocity "at the plane tangent to the outer edges of the cylinders in the first row" [27]. The uncertainty in the interfacial location for this experiment is expected to still fall within $\pm 0.11 \mathrm{~mm}$ which is half the size of the PIV interrogation window. The volume averaged slip velocity, $U_{v, s}$ are presented in Table 2 . To determine the shear rate at the interface, a curve of an adjusted coefficient of determination of more than 0.99 , was fitted to at least six $U$ data points located at the region close to the interface. A differentiation of the curve was then performed in the direction of the free zone flow to obtain the shear rate at the interface $\dot{\gamma}_{v}\left(=\mathrm{dU}_{\mathrm{v}} /\left.\mathrm{dy}\right|_{\mathrm{y}=0}\right)$. They are summarized in Table 2.

With $\mathrm{k}, \mathrm{I}_{\mathrm{s}^{\prime}} \mathrm{U}_{\mathrm{max}} \mathrm{U}_{\mathrm{d}}, \mathrm{U}_{\mathrm{s}^{\prime}}$ and $\dot{\gamma}_{v}$ determined, the interfacial flow could then be investigated using the dimensionless groupings $U_{v, s} / U_{v, \text { max }}$ and $U_{v^{\prime} s} /\left(\dot{\gamma}_{v} k^{05}\right)$. The line averaged dimensionless velocity defect $U_{\mathrm{l}, \mathrm{s}}-\mathrm{U}_{\mathrm{d}} /\left(\dot{\gamma} \mathrm{k}^{0.5}\right)$ is also characterized to compare with previous work. The total uncertainties associated with the volume averaged dimensionless $\mathrm{U}_{\mathrm{s}} / \mathrm{U}_{\max }$ are estimated to be $3 \%$ for $\phi=0.03,0.06$, and $5 \%, 8 \%$ and $10 \%$ for $\phi=0.12,0.22$ and 0.49 , respectively. The total uncertainties of $\mathrm{U}_{s} /\left(\dot{\gamma} \mathrm{k}^{0.5}\right)$ are approximately $5 \%$ for $\phi=0.06$, and $8 \%, 12 \%$ and $14 \%$ for $\phi=0.12,0.22$ and 0.49 respectively. The uncertainties of $\mathrm{U}_{\mathrm{l}, \mathrm{s}}-\mathrm{U}_{\mathrm{d}} /\left(\dot{\gamma} \mathrm{k}^{0.5}\right)$ are also estimated to be $8 \%$ for $\phi=0.06$, and $11 \%, 13 \%$ and $15 \%$ for $\phi=0.12,0.22$ and 0.49 , respectively. All of these uncertainties are at a confidence level of $95 \%$.

It is clear from Fig. 7a that as $\phi$ is increased from 0.06 to $0.49 \mathrm{U}_{\mathrm{v}, \mathrm{s}} / \mathrm{U}_{\mathrm{v}, \max }$ decreases by over $50 \%$. Agelinchaab et al. [23] observed a decreasing trend of $U_{s} / U_{\max }$ for their porous medium models reported, even though their porous media models were three-dimensional and filling fractions were 0.28 and 0.56 . This shows that generally, the decreasing trend of $U_{v, s} / U_{v, \max }$ with $\phi$ is associated with an increase in the resistance in flow through the porous medium.

A comparison of $U_{v, s} / U_{v, \max }$ ratios in Table 2 shows that the staggered arrays are about $15 \%$ less than nonstaggered arrays. However, $U_{v, s} / U_{v, \max }$ ratios of square and circular rods are similar. This result implies that for models of $\phi=0.12$, the arrangement of the models is of more importance at the slip, than the shape of the rods. Staggered arrays will yield smaller slip velocities than the non-staggered arrays. This seems reasonable as staggered models would intuitively offer greater resistance to flow at the interface.

The dependence of the dimensionless slip velocity $\mathrm{U}_{\mathrm{v} r} /\left(\dot{\gamma}_{v} \mathrm{k}^{0.5}\right)$ on $\phi$ is also shown in Fig. 7b. The parameter $\mathrm{U}_{\mathrm{v} / \mathrm{s}} /\left(\dot{\gamma}_{v} \mathrm{k}^{0.5}\right)$ generally increases by $32 \%$ as $\phi$ increases from 0.06 to 0.49 . This illustrates the prominent influence local conditions of the porous medium conditions on $\mathrm{U}_{\mathrm{v} / \mathrm{s}}$. This is however in contrast to previous results $[23,24]$, where $\mathrm{U}_{\mathrm{s}} /\left(\dot{\gamma} \mathrm{k}^{0.5}\right)$ was found to be independent of $\phi$. The difference shows that the type of porous medium affects the dimensionless parameter $\mathrm{U}_{v^{\prime} s} /\left(\dot{\gamma}_{v} \mathrm{k}^{0.5}\right)$, as expected. To better understand the trend of this dimensionless slip velocity however, the ratio $U_{v^{\prime} s} / \dot{\gamma}_{v}$ (as shown in Table 2) should be examined. This ratio may be interpreted as a measure of the screening length [27]. A comparison of the ratio indicates that $\mathrm{U}_{v^{\prime} s} / \dot{\gamma}_{v}$ decreases with increase in $\phi$. Thus, the increase in $U_{v r s} /\left(\dot{\gamma}_{v} \mathrm{k}^{0.5}\right)$ with $\phi$ suggest that using $\mathrm{k}^{0.5}$ to approximate the screening length for such a flow becomes less meaningful as the $\phi$ increases. 

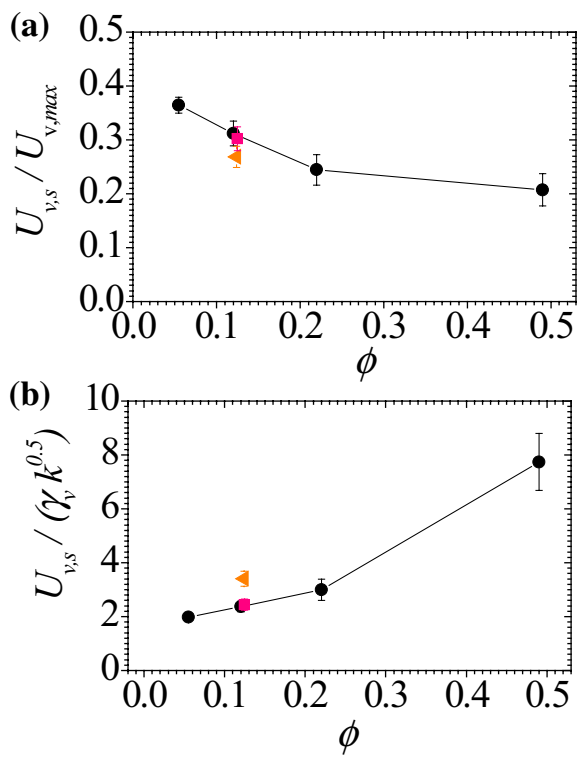

(c)

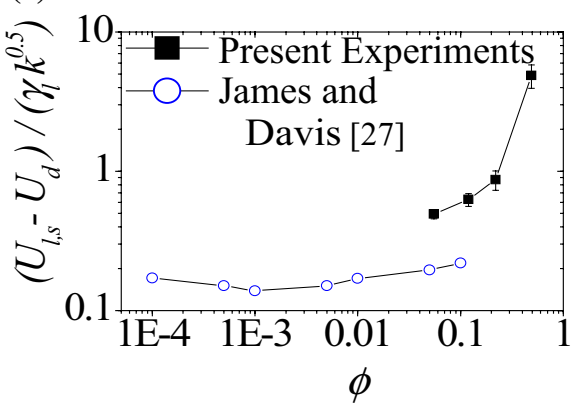

Fig. 7 Profiles of a $U_{v, s} / U_{\max } \mathbf{b} U_{s} /\left(\dot{\gamma} k^{0.5}\right)$; $\mathbf{c}$ line-averaged dimensionless slip velocity defect $\left(U_{l, s}-U_{d}\right) / \dot{\gamma}_{1} k^{0.5}$ values to compare with results [27] wherein $\mathrm{h} / \mathrm{H}=0.75$ and $\mathrm{H} / \mathrm{l}$ and $\mathrm{h} / \mathrm{l}$ are kept constant at 20 and 5 respectively. The present results are for test cases where $\mathrm{h} / \mathrm{H}=0.75$, varying $\mathrm{H} / \mathrm{l}$ and $\mathrm{h} / \mathrm{I}$ ratios

As shown in Fig. 7b, the arrangement of the rods, affects the value of the dimensionless $U_{v ' s} /\left(\dot{\gamma}_{v} \mathrm{k}^{0.5}\right)$. Accordingly, $\mathrm{U}_{\mathrm{v}, \mathrm{s}} /\left(\dot{\gamma}_{v} \mathrm{k}^{0.5}\right)$ is observed to vary with the staggered and staggered arrays of rods. The dimensionless slips for the staggered the model are at least 1.4 times that of nonstaggered arrays. The ratio $U_{s} / \dot{\gamma}$ shows that the penetration depth of the staggered model is $84 \%$ of the nonstaggered model. For the rod shapes, results of Fig. 7 and Table 2 show that the depths of penetration of the free zone flow into the porous medium is similar in both circular and square.

James and Davis [27] analyzed slow flow through and over two-dimensional model porous media of filling fraction of 0.75 . Although their study had $\phi \leq 0.10$, it is the most comprehensive in the literature available for some form of comparison. This is done in Fig. 7c for circular rods of non-staggered arrangement. However due to differences in $\mathrm{h} / \mathrm{I}$ and $\mathrm{H} / \mathrm{I}$ ratios (which James and Davis
[27] have showed to be important factors for the interfacial flow even for porous media of the same $h / H)$, the two results are not expected to be the same. As a result, the deviation between the line averaged dimensionless slip velocity defects $\left(U_{l, s}-U_{d}\right) / \dot{\gamma}_{l} k^{0.5}$ of the present results and that of James and Davis [27] are about 120\% even at $\phi=0.06$, where the solid volume fractions of the two studies coincide. It is also pointed out that although the twodimensional porous media results of Sahraoui and Kaviany [10] may not be appropriately compared with the present work due to differences in $\mathrm{h} / \mathrm{H}$, they also reported that the slip coefficient increases with porosity. This interpreted in the present results, means $\left(U_{l, 5}-U_{d}\right) / \dot{\gamma}_{l} k^{0.5}$ increases with $\phi$ as shown in Fig. 7c.

\subsection{Interfacial flow: verifications of formulations and prediction}

To close the present discussion, some proposed formulations of interfacial flow are verified using present data. An attempt is also made to predict the flow at the interface.

The Brinkman equation is the first formulation to be verified. Two models $[4,28]$ of its apparent viscosity $\mu^{\prime}$ are utilized, namely, $\mu / \mu^{\prime}=1$ and $\mu / \mu^{\prime}=\alpha^{2}$. To verify the applicability of these models, volume averaged data from the present experiments are compared in Fig. 8. Predictions from the use of the Brinkman equation are based on the following volume averaged form of the solution of the Brinkman equation valid in the porous medium [28].

$U_{v}(y)=U_{d}+\left(U_{v, s}-U_{d}\right) \exp \left[\frac{y}{\left(k \mu^{\prime} / \mu\right)^{0.5}}\right]$

It should be noted that if $\mu / \mu^{\prime}=a_{v}^{2}$, differentiating Eq. (14) once with respect to $y$, and evaluating it at $y=0$ should give

$\frac{d U_{v}}{d y}=\frac{\alpha_{v}}{k^{0.5}}\left(U_{v, s}-U_{d}\right)$

Equation (15) is a modified form of the BJC, accounting for continuity in both velocity and shear at the interface. Thus using $\mu / \mu^{\prime}=a_{v \prime}^{2}$ Eq. (14) should predict the velocity distribution. For the specific cases of $\mu / \mu^{\prime}=a_{v}^{2}$ used in Fig. 8, $a_{v}$ was derived from Eq. (15) using experimental results obtained for the respective test cases. Figure 8 shows that apart from the singular case of agreement between experiments and the Brinkman model (i.e. $\mu / \mu^{\prime}=1$ ) for $\operatorname{RdN}$ $\phi_{6}$ (resulting in an adjusted coefficient of determination of 0.97 ) the two models of the Brinkman equation give wrong predictions.

To verify the utility of the OWC with respect to this work and to provide some data regarding its empirical 
Fig. 8 Verifying the Brinkman equation using experimental data at the interfacial zone of a $\operatorname{RdN} \phi_{6}$ b $\operatorname{RdN} \phi_{12}$ c $\operatorname{RdN} \phi_{22}$ d $\mathrm{RdN} \phi_{49}$ e SqN $\phi_{12}$ and $\mathbf{f} \mathrm{RdSt}$ $\phi_{12}$ (a)

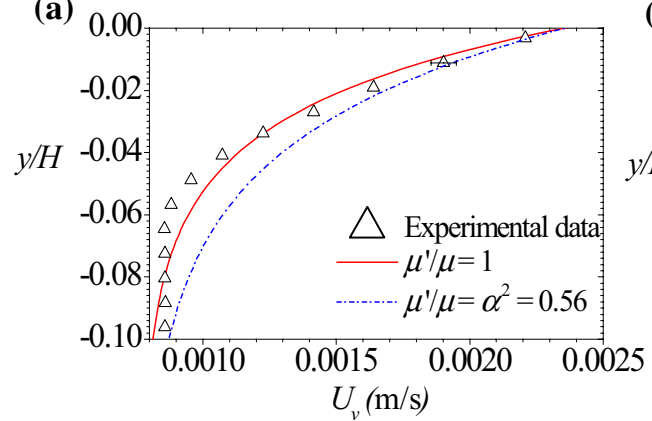

(c)

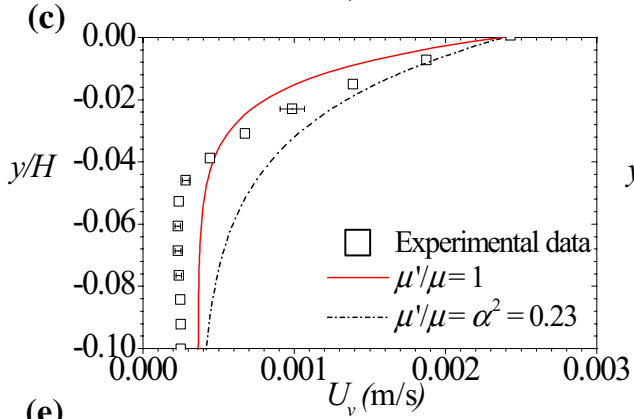

(e)

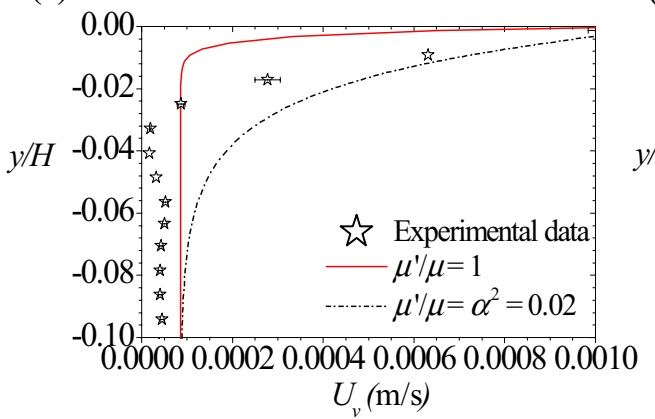

(b)

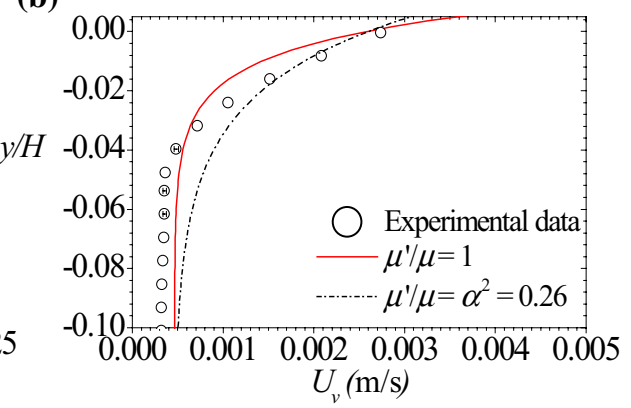

(d)

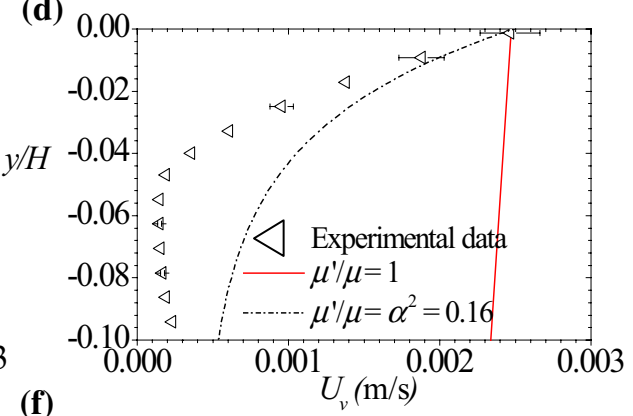

(f)

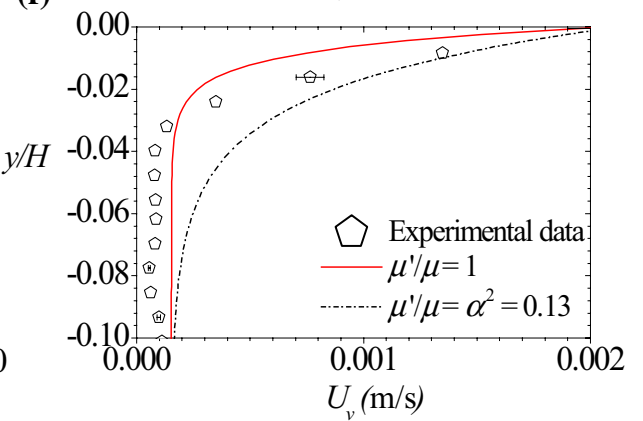

coefficient $\beta$, an additional up-scaling was performed at the interfacial region of the volume averaged data. This was done using a transverse length equal to twice the transition layer thickness $\delta_{1}$ within the porous medium. It should be noted that $\delta_{1}$ is defined in this work as the transverse distance taken for the $U_{v}$ to decay to $1.01 U_{d}$ with reference to the interfacial location [29]. The values are summarized are in Table 3. Included in Table 3 are the values of $\beta$. From the results, it may be observed that $\beta$ ranges over more than one order of magnitude as $\phi$ is varied from 0.06 to 0.49 . While this dependence is unclear it is independent of the shape of the rods. However, $\beta$ reduces significantly (by about $30 \%$ for $\phi=0.12$ ) when arrays are changed from inline to staggered array. One clear limitation of the OWC in this work is that it results in a jump in shear at the interface, which is clearly not the case in the present results.

To provide an alternative to predict the interfacial flow curve-fitting techniques were applied to the experimental data at the interfacial region. For the present results, the interfacial flow was found to be well described by the
Table 3 Summary of values pertaining to prediction of interfacial flow

\begin{tabular}{lllll}
\hline Name & $\delta_{1}(\mathrm{~mm})$ & $\beta$ & $\mathrm{R}^{2}$ & $\alpha_{v}$ \\
\hline $\operatorname{RdN} \phi_{6}$ & 14.0 & 7.35 & 0.998 & 0.75 \\
$\operatorname{RdN} \phi_{12}$ & 4.2 & 0.57 & 0.997 & 0.51 \\
$\operatorname{RdN} \phi_{22}$ & 3.4 & 0.44 & 0.998 & 0.36 \\
$\operatorname{RdN} \phi_{49}$ & 2.8 & 0.76 & 0.998 & 0.14 \\
$\mathrm{SqN} \phi_{12}$ & 4.1 & 0.59 & 0.996 & 0.48 \\
$\operatorname{RdSt} \phi_{12}$ & 4.2 & 0.43 & 0.990 & 0.36 \\
\hline
\end{tabular}

following dose response curve, with an adjusted coefficient of determination of 0.99 and above (as shown in Fig. 9 and in Table 3)

$U_{v}(y)=A+\frac{B-A}{1+10^{(D-y) p}}$

Here, $A, B, D$, and $p$ are respectively the asymptote as $y \rightarrow$ $-\infty$, the asymptote as $y \rightarrow \infty$, the transverse component of the center of the curve, and the hill slope of the curve. The 
curve fits are shown for all test conditions in Fig. 9. The shear rate of the flow at the interface yields

$\left.\frac{d U_{v}}{d y}\right|_{y=0+}=\frac{(B-A) 10^{D p}}{\left(1+10^{D p}\right)^{2}}[p \ln 10]$

This equation is the boundary condition at the interface between the porous medium and free zone flow of the configuration studied. As mentioned earlier, as $\mathrm{y} \rightarrow-\infty$ in Eq. (16), $U=A$ which is equivalent to the seepage velocity $U_{d}$. Furthermore, at $\mathrm{y}=0$ (i.e. at the interface),

$U_{v}(0)=U_{v, s}=A+\frac{B-A}{1+10^{D p}}$

The boundary condition in Eq. (17) may therefore be interpreted as a fulsome form of the BJC as the velocity defect is

$U_{v, s}-U_{d}=\frac{(B-A)}{1+10^{D p}}$

Thus rearranging Eq. (18) in the form of the BJC, it may be shown that $\left.\frac{d U_{v}}{d y}\right|_{y=0+}=\left[\frac{10^{D p}}{\left(1+10^{D p}\right)} p \ln 10\right]\left(U_{v, s}-U_{d}\right)=\frac{\alpha_{v}}{k^{0.5}}\left(U_{v, s}-U_{d}\right)$

The coefficient $a_{v}$ is equivalent to the slip coefficient of the $B J C$, and it is provided in Table 3 . The values show that $a_{v}$ decreases by $\sim 50 \%$ with $\phi$ increasing from 0.06 to 0.49 . While $a_{v}$ is independent of the shape of the rods, it differs by $\sim 30 \%$ between staggered and non-staggered models. Indeed, $a_{v}$ of the staggered model of $\phi=0.12$ is equivalent to the non-staggered model of $\phi=0.22$. Unlike the coefficients $\beta, a_{v}$ ranges over less than one order of magnitude.

While likening Eq. (20) to the BJC, it is important to remark that the BJC was based on a simplified model in which the shear rate within the porous medium was not considered. This therefore resulted in a description in which there was a discontinuity in velocity, and a jump in the shear at the interface. Equation (20) corrects this deficiency by accounting for continuities in velocity and stress at the interface. The form of this boundary condition indicates the complex nature of the interfacial flow.
Fig. 9 Experimental data at the interfacial zone fitted to data points of $\mathbf{a} \operatorname{RdN} \phi_{6} \mathbf{b} \operatorname{RdN} \phi_{12}$ c $\operatorname{RdN} \phi_{22}$ d RdN $\phi_{49}$ e $\operatorname{SqN} \phi_{12}$ and $\mathbf{f} \operatorname{RdSt} \phi_{12}$
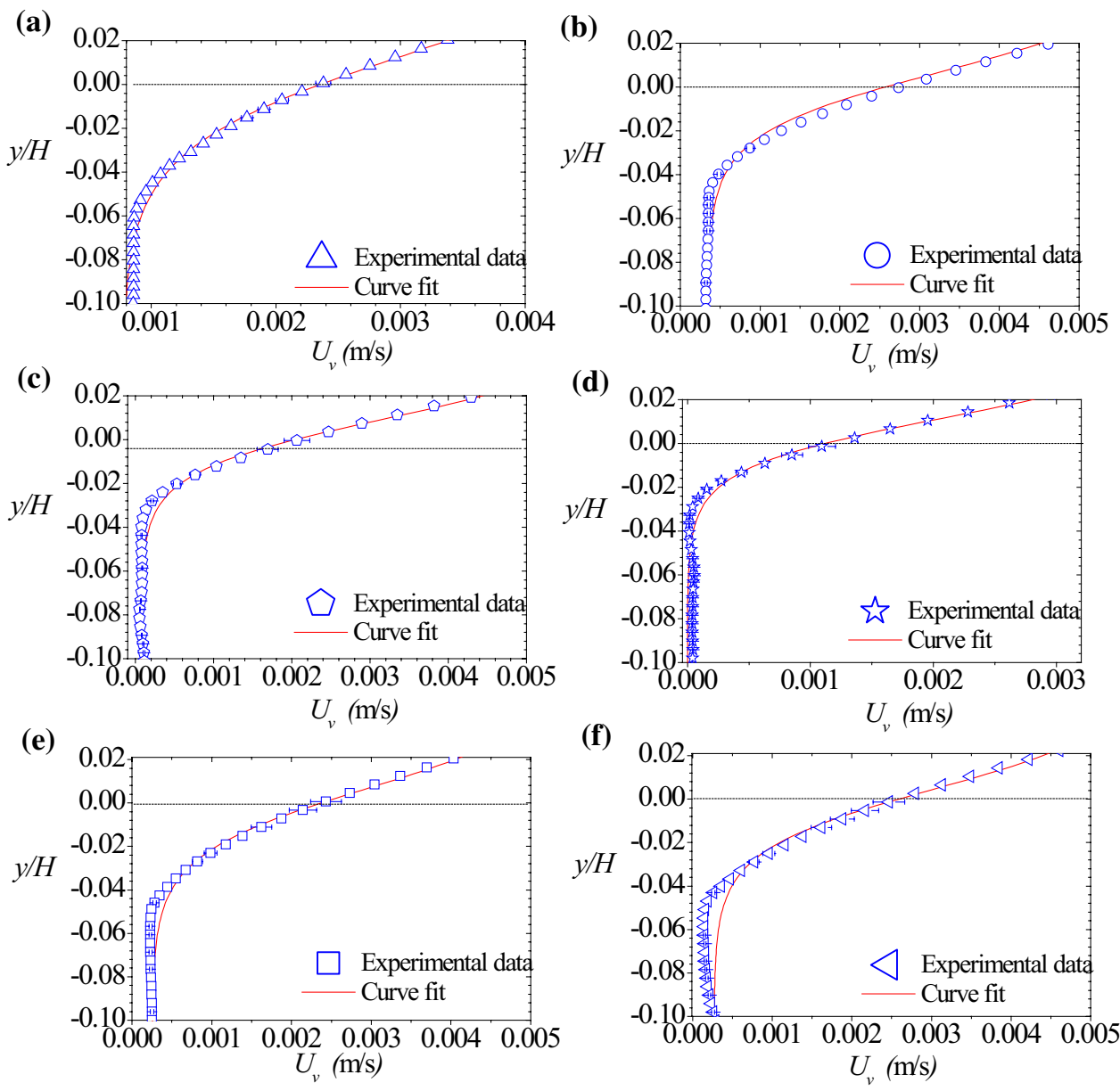


\section{Conclusions}

In this work, results of PIV measurements of flow through and over two-dimensional model porous media have been presented and analyzed. The following conclusions may be drawn from the study:

(a) For the low Reynolds number flow tested, transverse velocities may be neglected when compared with the local maximum streamwise velocities irrespective of the solid volume fraction, the mode of arrangement, and the shape of the rod shape.

(b) For the media tested, the greater percentage of the bulk flow is channelled through the free zone. This ranges from 75 to $98 \%$ as $\phi$ increases from 0.06 to 0.49 . The percentage flow distributions, being bulk measurements are nearly independent of the shape and arrangement of rods.

(c) At the interface, the ratio of slip velocity to the corresponding maximum velocity decreases gradually from 0.36 to 0.25 as $\phi$ increases from 0.06 to 0.49 . Staggered arrays are about $15 \%$ less that of non-staggered arrays, while square and circular rods record similar ratios.

(d) Other dimensionless velocities related to the interfacial shear rate show that the depth of penetration is reduced by at least half when $\phi$ increases from 0.06 to 0.49 . On one hand, the penetrations are reduced by at least $15 \%$ when non-staggered arrays are staggered. On the other hand, the penetrations are independent of the shape of the rods.

(e) The boundary condition resulting from the formulation predicting the interfacial flow of the present results conditions is a modification of that of Beavers and Joseph [8]. Its related slip coefficient varies from 0.75 to 0.36 as $\phi$ increases from 0.06 to 0.49 . It is dependent on the mode of arrangement of the rods, but independent of the shape of the rods.

As the present work only considers only three parameters of the flow problem, the conclusions of this work are not general enough. It also remains to be determined how the flow formulation in Eq. (16) is connected to any momentum equation such as the Brinkman equation, or the Navier-Stokes equation. Regardless, this work provides considerable insight into the flow phenomena through and over two-dimensional porous media and may be used to validate theoretical models. Moreso, the detailed interfacial boundary condition presented herein will serve as a useful tool for analyzing interfacial flows.

Acknowledgements The immense inputs of Profs D.W. Ruth and M.F. Tachie, and the financial support of this work by the Natural Sciences and Engineering Research Council of Canada (NSERC) through research grants, are gratefully acknowledged.

\section{Compliance with ethical standards}

Conflict of interest The author declares that he has no conflict of interest.

\section{References}

1. Shavit U (2009) Special issue: transport phenomena at the interface between fluid and porous domains a preface. Transp Porous Med 78:327-540

2. Nield DA, Bejan A (2006) Convection in porous media. Springer, New York

3. Chandesris M, Jamet D (2007) Boundary conditions at a fluidporous interface: an a priori estimation of the stress jump coefficients. Int J Heat Mass Transf 50:3422-3436

4. Brinkman HC (1947) A calculation of the viscous force exerted by a flowing fluid on a dense swarm of particle. Appl Sci Res Sect A 1:27-34

5. Larson RE, Higdon JJL (1986) Microscopic flow near the surface of two-dimensional porous media Part 1. Axial flow J Fluid Mech 166:449-472

6. Larson RE, Higdon JJL (1987) Microscopic flow near the surface of two-dimensional porous media Part 2. Trans Flow J Fluid Mech 178:119-136

7. Almalki WS, Hamdan MH (2016) Investigations in effective viscosity of fluid in a porous medium. Int J Eng Res Appl 6(4):41-51

8. Beavers GS, Joseph DD (1967) Boundary conditions at a naturally permeable wall. J Fluid Mech 30:197-207

9. Ochoa-Tapia JA, Whitaker S (1995) Momentum-transfer boundary between a porous medium and a homogenous fluid-I. Theoretical development. Int J Heat Mass Transf 38:2635-2646

10. Sahraoui M, Kaviany M (1991) Slip and no-slip conditions at interface of porous, open media. ASME/JSME Therm Eng Proc 4:273-286

11. Wu Z, Mirbod P (2018) Experimental analysis of the flow near the boundary of random porous media. Phys Fluids 30:047103. https://doi.org/10.1063/1.5021903

12. Terzis A, Zarikos I, Weishaupt K, Yang G, Chu X, Helmig R, Weigand B (2019) Microscopic velocity field measurements inside a regular porous medium adjacent to a low Reynolds number channel flow. Phys Fluids 31:042001. https://doi. org/10.1063/1.5092169

13. Davis AMJ, James DF (2004) Penetration of shear flow into an array of rods aligned with the flow. Can J Chem Eng 82:1169-1174

14. Shams M, James DF, Currie IG (2003) The velocity field near the edge of a model porous medium. Exp Fluids 35:193-198

15. Tachie MF, James DF, Currie IG (2003) Velocity measurements of the shear flow penetrating a porous medium. J Fluid Mech 493:319-343

16. Arthur JK, Ruth DW, Tachie MF (2013) Porous medium flow and an overlying parallel flow: PIV interrogation area and overlaps, interfacial location, and depth ratio effects. Transp Porous Med 97:5. https://doi.org/10.1007/s11242-012-0107-9

17. Arthur JK (2012) Flow through and over porous media with or without inertial effects. Ph.D. thesis, University of Manitoba, Winnipeg, Manitoba, Canada. https://mspace.lib.umanitoba.ca/ bitstream/handle/1993/8599/Arthur_James.pdf?sequence $=1$

18. Raffel M, Willert C, Wereley S, Kompenhans J (2007) Particle image velocimetry: a practical guide, 2 nd edn. Springer, Berlin 
19. Prasad AK (2000) Particle image velocimetry. Curr Sci 79(1):51-60

20. Scarano F, Riethmuller ML (1999) Iterative multigrid approach in PIV image processing with discrete window offset. Exp Fluids 26(6):513-523

21. Coleman WH, Steele WG (1995) Engineering application of experimental uncertainty analysis. AIAA J 33:1888-1896

22. Forliti DJ, Strykowski PJ, Debatin K (2000) Bias and precision errors of digital particle image velocimetry. Exp Fluids 28(5):436-447

23. Agelinchaab M, Tachie MF, Ruth DW (2006) Velocity measurement of flow through a model three-dimensional porous medium. Phys Fluids 18:017105-1

24. Arthur JK, Ruth DW, Tachie MF (2009) PIV measurements of flow through a model porous medium with varying boundary conditions. J Fluid Mech 629(1):343-374

25. Jackson GW, James DF (1986) The permeability of fibrous porous media. Can J Chem Eng 64:364-374
26. Hellou M, Martinez J, El Yazidi M (2004) Stokes flow through microsctructural model of fibrous media. Mech Res Commun 31(1):97-103

27. James DF, Davis AMJ (2001) Flow at the interface of a model fibrous medium. J Fluid Mech 426:47-71

28. Neale G, Nader W (1974) Practical significance of Brinkman's extension of Darcy's law-coupled parallel flows within a channel and a bounding porous-medium. Can J Chem Eng $52: 475-478$

29. Morad RZ, Khalili A (2009) Transition layer thickness in a fluidporous spherical beads. Exp Fluids 46:323-330

Publisher's Note Springer Nature remains neutral with regard to jurisdictional claims in published maps and institutional affiliations. 